\title{
Sampling nucleotide diversity in cotton
}

\author{
Allen Van Deynze*1, Kevin Stoffel ${ }^{1}$, Mike Lee ${ }^{1}$, Thea A Wilkins ${ }^{2}$, \\ Alexander Kozik ${ }^{3}$, Roy G Cantrell ${ }^{4}$, John Z Yu${ }^{5}$, Russel J Kohel ${ }^{5}$ and \\ David M Stelly ${ }^{6}$
}

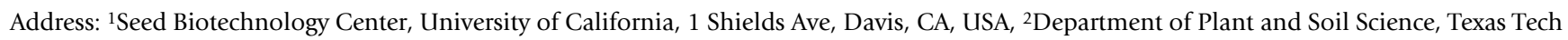 \\ University, Experimental Sciences Building, Room 215, Mail Stop 3132, Lubbock, TX 79409-3132, USA, ${ }^{3}$ Genome and Biomedical Sciences \\ Facility, University of California, 1 Shields Ave, Davis, CA, USA, ${ }^{4}$ Monsanto, 1800 N. Lindbergh Blvd, St Louis, MO 63167, USA, 5USDA-ARS, \\ Southern Plains Agricultural Research Center, 2881 F\&B Road, College Station, TX 77845, USA and ${ }^{6}$ Department of Soil and Crop Sciences, Texas \\ A \& M University, College Station, TX 77843, USA \\ Email: Allen Van Deynze* - avandeynze@ucdavis.edu; Kevin Stoffel - kmstoffel@ucdavis.edu; Mike Lee - mklee@ucdavis.edu; \\ Thea A Wilkins - thea.wilkins@ttu.edu; Alexander Kozik - akozik@atgc.org; Roy G Cantrell - roy.cantrell@monsanto.com; \\ John Z Yu - john.yu@ars.usda.gov; Russel J Kohel - kohel@qutun.tamu.edu; David M Stelly - stelly@tamu.edu \\ * Corresponding author
}

Published: 20 October 2009

BMC Plant Biology 2009, 9:125 doi:10.1 186/147/-2229-9-125
Received: 30 September 2008

Accepted: 20 October 2009

This article is available from: http://www.biomedcentral.com/I47/-2229/9/125

(c) 2009 Van Deynze et al; licensee BioMed Central Ltd.

This is an Open Access article distributed under the terms of the Creative Commons Attribution License (http://creativecommons.org/licenses/by/2.0), which permits unrestricted use, distribution, and reproduction in any medium, provided the original work is properly cited.

\begin{abstract}
Background: Cultivated cotton is an annual fiber crop derived mainly from two perennial species, Gossypium hirsutum L. or upland cotton, and G. barbadense L., extra long-staple fiber Pima or Egyptian cotton. These two cultivated species are among five allotetraploid species presumably derived monophyletically between G. arboreum and G. raimondii. Genomic-based approaches have been hindered by the limited variation within species. Yet, population-based methods are being used for genome-wide introgression of novel alleles from $G$. mustelinum and $G$. tomentosum into $G$. hirsutum using combinations of backcrossing, selfing, and inter-mating. Recombinant inbred line populations between genetics standards TM-I, (G. hirsutum) $\times$ 3-79 (G. barbadense) have been developed to allow high-density genetic mapping of traits.
\end{abstract}

Results: This paper describes a strategy to efficiently characterize genomic variation (SNPs and indels) within and among cotton species. Over 1000 SNPs from 270 loci and 279 indels from 92 loci segregating in $G$. hirsutum and $G$. barbadense were genotyped across a standard panel of 24 lines, 16 of which are elite cotton breeding lines and 8 mapping parents of populations from six cotton species. Over 200 loci were genetically mapped in a core mapping population derived from TM-I and 3-79 and in G. hirsutum breeding germplasm.

Conclusion: In this research, SNP and indel diversity is characterized for 270 single-copy polymorphic loci in cotton. A strategy for SNP discovery is defined to pre-screen loci for copy number and polymorphism. Our data indicate that the $A$ and $D$ genomes in both diploid and tetraploid cotton remain distinct from each such that paralogs can be distinguished. This research provides mapped DNA markers for intra-specific crosses and introgression of exotic germplasm in cotton. 


\section{Background}

The cotton family consists of 45 diploid species $(2 \mathrm{n}=2 \mathrm{x}$ $=26$ ) representing eight genome groups $(\mathrm{A}, \mathrm{B}, \mathrm{C}, \mathrm{D}, \mathrm{E}, \mathrm{F}$, $\mathrm{G}, \mathrm{K})$ and five AD allotetraploid species $(2 \mathrm{n}=4 \mathrm{x}=52)$ that are inter-crossable to various degrees. Cultivated cotton is an annual fiber crop derived mainly from two perennial species, Gossypium hirsutum L. or upland cotton, and G. barbadense L., extra long-staple fiber Pima or Egyptian cotton. These two cultivated species are among five allotetraploid species presumably derived monophyletically from a single polyploidization event that occurred 12 MYA between ancestors most closely represented today by G. arboreum (A2 genome) and G. raimondii (D5 genome). Breeding of cotton is primarily focused on intraspecific crosses to introduce transgenic traits and to improve baseline lint yield and quality [1], although significant advances can be made through inter-specific introgression [2-4]. The relatively recent speciation of tetraploid cotton affords opportunities to transfer novel traits between species, but also amplifies the challenge of maintaining the high yields and quality requisite of commercial products. G. tomentosum (AD3) and G. mustelinum (AD4) are rich sources of novel traits that are currently being mined to improve cotton agronomy and fiber. DNA markers can provide means of detecting, manipulating and identifying genes associated with desirable agronomic and quality traits within breeding programs, as well as novel alleles from wide crosses. They have shown to be useful in accelerating the transfer of novel traits into elite backgrounds, particularly when these markers have been placed on genetic maps [5].

The most extensive genetic maps in cotton have been derived mainly from wide crosses between the two ADgenome species G. hirsutum and G. barbadense [6-8]. Interspecific maps also exist between $G$. hirsutum and G. tomentosum [9]. DNA markers have been oriented to chromosomes and used to establish co-linearity among genomes and species using radiation hybrids and hypo-aneuploid $\mathrm{F}_{1}$ hybrids, available for most chromosomes. In total, approximately 5,000 DNA markers have been mapped. These were derived from approximately 3,300 restriction fragment length polymorphisms (RFLPs), 700 amplified fragment length polymorphisms (AFLPs), 1,000 simple sequence repeats (SSRs), and 100 single nucleotide polymorphisms (SNPs) [10]. Furthermore, 2,584 sequencetagged site (STS) loci are mapped in an AD genome and 1,014 in and the D genome $[8,11]$. An EST-SSR map with 1,017 loci is also available [12]. Only a few low resolution intra-specific maps that focus on specific traits exist due to the low level of polymorphism within a species and the paucity of cost-efficient markers available to be used in breeding programs.
The vast majority of markers in cotton that are useful in breeding are as SSRs. Over 8,000 pairs of SSR primers are identified in cotton from G. arboreum, G. raimondii and $G$. hirsutum [13]. The frequency of polymorphism within species has been reported to be limited to $11 \%$ [14]. Informative, abundant, high-throughput markers associated with genes such as SNPs or insertion/deletions (indels) are desirable for both breeding and genetic analyses. Expressed genes are available as templates to study variation. In, G. arboreum, 24,597 non-redundant transcripts are available; with 27,355 in G. raimondii; and 63,138 in G. hirsutum [15]. The goal of the current project was to design a strategy to efficiently identify and characterize SNP markers that are useful to manipulate and transfer novel alleles to breeding germplasm in cotton. Different DNA templates were evaluated for their specificity to amplify single-copy loci, and polymorphism within and among species, with emphasis on cultivated cotton. We show that single-copy loci can be efficiently amplified in cotton despite redundancy conferred by its allopolyploid origin, and that they can be mapped to specific genomes. The information can be queried in the Cotton Marker Database which has been modified for presentation of SNP data [16]

\section{Results and Discussion Defining optimum regions to sample for SNPs in cotton} Our goal was to define and optimize a SNP discovery strategy in cotton that exploits current genomic resources. The disomic polyploid nature of cotton poses a particular challenge in that most loci are duplicated, and breeding germplasm is derived from a relatively narrow genetic base $[17,18]$. A re-sequencing strategy developed by our lab was modified to anchor and screen DNA primer pairs that amplify single-copy, polymorphic regions of the genome relevant to current breeding germplasm that can also be used for introgression of novel alleles from exotic germplasm (See Materials and Methods and [19]). To address this, we empirically evaluated the proportion of single-copy sequences obtained from amplicons originating from different genomic regions, namely: BAC-end sequences, sequences flanking SSRs or predicted introns in ESTs, and sequences in the $3^{\prime}$ or 5 ' untranslated region of ESTs (Table 1). Although the majority (93\%, data not shown) of amplifiable primer sets showed single bands on agarose gels, SSCP analysis revealed that only 51\% and $40 \%$ of the primer pairs amplified single-copy sequences in G. arboreum and G. hirsutum, respectively. With SSCP analysis of single varieties, an amplicon from a singlecopy locus in homozygous state, whether from a diploid or tetraploid is expected to display only two bands, one from sense and one anti-sense strands. As the lines being assayed were near-homozygous, only loci with two SSCP bands were carried forward. 
Table I: Summary statistics for SNPs and indels among 24 lines of cotton derived from different DNA templates.

\begin{tabular}{|c|c|c|c|c|c|c|c|c|c|c|c|}
\hline $\begin{array}{l}\text { DNA } \\
\text { template }\end{array}$ & $\begin{array}{r}\text { Primer } \\
\text { pairs } \\
\text { designed }\end{array}$ & $\begin{array}{r}\text { Amplified } \\
\text { primer } \\
\text { pairs (\%) }\end{array}$ & $\begin{array}{r}\text { Single-copy } \\
\text { loci }(\%)^{\prime}\end{array}$ & $\begin{array}{l}\text { Polymorphic } \\
\text { SNP loci (\%) }\end{array}$ & SNPs & $\begin{array}{l}\text { SNPs/ } \\
\text { locus }\end{array}$ & $\begin{array}{r}\text { bp/ } \\
\text { SNP2 }^{2,3}\end{array}$ & $\begin{array}{r}\text { Polymorphic } \\
\text { indel } \\
\text { loci }(\%)^{2}\end{array}$ & Indels & $\begin{array}{r}\text { Indels/ } \\
\text { locus }\end{array}$ & $\begin{array}{r}\text { bp/ } \\
\text { indel }^{2,3}\end{array}$ \\
\hline EST 3 ' end & 160 & $102(64)$ & $45(28,44)$ & $30(67)$ & 144 & 3.2 & 252 & $14(3 \mid)$ & 49 & 1.1 & 741 \\
\hline EST 5 ' end & 988 & $802(73)$ & $417(42,52)$ & 142 (34) & 417 & 1.0 & 807 & $37(9)$ & 109 & 0.3 & 3,087 \\
\hline $\cos$ & 576 & $523(91)$ & $201(35,38)$ & $56(28)$ & 296 & 1.5 & 548 & $20(10)$ & 70 & 0.3 & 2,317 \\
\hline Intron & 126 & 98 (78) & $44(35,45)$ & 17(39) & 46 & 1.0 & 772 & II (25) & 32 & 0.7 & 1,110 \\
\hline GSP & 48 & $29(60)$ & $23(48,79)$ & $11(48)$ & 40 & 1.7 & 464 & $5(22)$ & 12 & 0.5 & 1,547 \\
\hline SSR & 52 & $46(88)$ & $21(40,46)$ & $14(67)$ & 62 & 3.0 & 273 & $5(24)$ & 7 & 0.3 & 2,421 \\
\hline BAC & 24 & $7(29)$ & $0(0,0)$ & $\mathrm{n} / \mathrm{a}$ & $\mathrm{n} / \mathrm{a}$ & $\mathrm{n} / \mathrm{a}$ & $\mathrm{n} / \mathrm{a}$ & $\mathrm{n} / \mathrm{a}$ & $\mathrm{n} / \mathrm{a}$ & $\mathrm{n} / \mathrm{a}$ & $\mathrm{n} / \mathrm{a}$ \\
\hline Total & 1,974 & $\mathrm{I}, 607(8 \mathrm{I})$ & $75 I$ (47) & $270(36)$ & 1,005 & 1.3 & 603 & $92(12)$ & 279 & 0.4 & 2,172 \\
\hline
\end{tabular}

IFirst number is percent of designed; second is percent of amplified

${ }^{2}$ Relative to single-copy loci

${ }^{3}$ Adjusted to average contig length of 807 bp

The amplicon templates exhibited a range of amplification, copy number and polymorphism (Table 1). The low rate of amplification $(29 \%)$ achieved when primers were designed from BAC-end genomic sequence deterred us from using this as template for primer design. The relatively poor rate of success might have resulted from inadequate quality of BAC-end genomic sequence available at the time (Sept, 2004). Cotton genome-specific (GSP) amplicons, those derived from primers spanning a SNP between G. arboreum and G. raimondii, resulted in the highest percentage (48\%) of single-copy sequences (Table 1). Primer pairs neighboring genomic SSRs also resulted in a high percentage $(46 \%)$ of single-copy sequences, but the relatively small amount of sequence flanking each SSR, 150-200 bp, limited the number of nucleotides available within an amplicon for SNP discovery. Amplicons that encompass introns or are derived from a conserved orthologous set (COS) of sequences that are single copy in Arabidopsis and cotton ESTs showed excellent amplification. However, as their primers are anchored in coding regions, they are likely to be the most conserved across paralogs, as indicated by the relatively low proportion (38\% for introns and $45 \%$ for COS primers) of singlecopy sequences. The 5' UTR of ESTs are the most abundant in the EST dataset and resulted in the highest proportion of amplicons with single-copy loci overall (42\%), next to the GSPs that became available only late in the project. It is important to note that the ESTs used were created by capturing sequences from the 3' UTR and sequencing from either the $3^{\prime}$ or $5^{\prime}$ end. Consequently, the $5^{\prime}$ sequences may not necessarily represent the 5 ' terminus of genes if clones were not full length. As a comparison, Chee et al. (2004) used sequences from G. arboreum to amplify G. hirsutum [20]. The authors reported that 33\% $(16 / 89)$ of primer pairs yielded amplicons from singlecopy loci, which is similar to the current results (average $38 \%$ across EST-derived amplicons, Table 1). This indi- cates the high level of homology of exons between homoeologous genomes in tetraploid cotton. The results of this study indicate that although genomic regions harbor genetic diversity, strategies must be developed to ensure that allelic diversity and not diversity between homoeologs and paralogs are being assayed.

\section{Diversity of genomic regions}

To study the diversity of cotton, we tested different DNA templates to select the optimum regions that will yield single-copy, yet polymorphic amplicons from PCR. Of the 1,974 primer pairs designed, $8 \%$ were from the 3 ' end; $50 \%$ 5'end; $29 \%$ COS; $6 \%$ intron; $3 \%$ SSR; $2 \%$ genomespecific primers; and $1 \%$ genomic (BAC-end) primers. Eighty-one percent successfully amplified DNA in a single G. arboreum and G. hirsutum line and $47 \%$ produced single-copy amplicons based on SSCP gels (Table 2). The relatively high amplification rate across species from primers designed mainly from diploid species confirms the transferability of markers across species as indicated in previous studies [21-25]. To pre-screen primer pairs for polymorphism, DNA pools representing increasing diversity within G. hirsutum and G. barbadense germplasm and among these two species were amplified and sequenced. Pool 1 represents G. hirsutum; Pool 2, G. hirsutum/G. hirsutum race yucatenense; and Pool 3, G. hirsutum and G. barbadense (Table 2). Polymorphism within pools was identified as heterozygotes, whereas polymorphism among pools was identified as differences in homozygous alleles. Polymorphic loci were then sequenced individually in the forward and reverse directions in 24 lines (Table 2). Forty-nine percent of primers with single-copy loci were polymorphic within or among pools. Overall, the pools represent the two cultivated species in the United States, G. hirsutum and G. barbadense, which explains the high polymorphism (percent polymorphic loci) and SNP frequency (bp/SNP) compared to within 
Table 2: Germplasm panel sequenced for SNP or indel discovery.

\begin{tabular}{|c|c|c|c|c|}
\hline Line' $^{\prime}$ & Genome & Description & CMD panel & Pool2 \\
\hline Acala Maxxa & {$[A D]_{1}$} & California Upland cotton and BAC donor & Yes & Pool 2, 3 \\
\hline AHA 6-I-4 & {$[A D]_{1}$} & Upland cotton & & \\
\hline Auburn 623RNR & {$[\mathrm{AD}]_{\mathrm{I}}$} & Upland cotton & & \\
\hline Coker 312 & {$[\mathrm{AD}]_{\mathrm{I}}$} & Upland cotton & & \\
\hline Deltatype Webber & {$[A D]_{1}$} & Upland cotton & & \\
\hline DPL 458BR & {$[\mathrm{AD}]_{1}$} & Upland cotton & Yes & Pool I \\
\hline Fibermax 832 & {$[A D]_{1}$} & Upland cotton & Yes & Pool I \\
\hline Paymaster $1218 \mathrm{BR}$ & {$[A D]_{1}$} & Upland cotton & Yes & \\
\hline PD-I & {$[A D]_{1}$} & Upland cotton & & Pool 2 \\
\hline Sealand 542 & {$[A D]_{1}$} & Upland cotton & & Pool I \\
\hline Stoneville 20 & {$[A D]_{1}$} & Upland cotton & & \\
\hline Stoneville 4892BR & {$[A D]_{1}$} & Upland cotton & Yes & Pool I \\
\hline Tamcot Sphinx & {$[A D]_{1}$} & Upland cotton & & Pool 2 \\
\hline Tidewater Seabrooks & {$[A D]_{1}$} & Upland cotton & & \\
\hline TM-I & {$[A D]_{1}$} & Genetic standard (BAC donor/RI parent) & Yes & Pool 3 \\
\hline Wilt Acala 1517 & {$[A D]_{1}$} & California Upland cotton & & \\
\hline TX 2094 & {$[A D]_{1}$} & G. hirsutum race yucatanense & & Pool 2 \\
\hline $3-79$ & {$[\mathrm{AD}]_{2}$} & Genetic standard (fiber QTLs/RI parent) & Yes & Pool 3 \\
\hline Pima S-6 & {$[\mathrm{AD}]_{2}$} & Pima germplasm breeding source & Yes & \\
\hline Pima S-7 & {$[\mathrm{AD}]_{2}$} & Pima germplasm breeding source & & Pool 3 \\
\hline G. tomentosum & {$[A D]_{3}$} & Introgression breeding source & Yes & \\
\hline G. mustelinum & {$[\mathrm{AD}]_{4}$} & Introgression breeding source & Yes & \\
\hline G. arboreum & $A_{2-8}$ & A-genome species representative & Yes & \\
\hline G. raimondii & $D_{5-3}$ & D-genome species representative & Yes & \\
\hline
\end{tabular}

IAll 12 members of the Cotton Marker Database Panel were included [13]

2Pools of 4 lines representing cotton germplasm were used to screen primer sets (see Materials and Methods)

Table 3: Species-specific statistics for Gossypium SNPs and indels.

\begin{tabular}{|c|c|c|c|c|c|c|c|c|c|c|c|}
\hline Species & $\mathbf{N}$ & $\begin{array}{l}\text { Loci } \\
\text { with } \\
\text { SNPs }\end{array}$ & $\begin{array}{r}\text { Number } \\
\text { of SNPs }\end{array}$ & $\begin{array}{l}\text { Loci with } \\
\text { SNPs (\%)' }\end{array}$ & $\begin{array}{l}\text { SNPs/ } \\
\text { locus' }\end{array}$ & $\begin{array}{r}\text { bp/ } \\
\text { SNPI,2 }\end{array}$ & $\begin{array}{l}\text { Lociwith } \\
\text { indels }\end{array}$ & $\begin{array}{l}\text { Number } \\
\text { of indels }\end{array}$ & $\begin{array}{l}\text { Loci with } \\
\text { indels (\%)' }\end{array}$ & $\begin{array}{l}\text { Indels/ } \\
\text { locus' }\end{array}$ & $\begin{array}{r}\text { Bases/ } \\
\text { indel } 1,2\end{array}$ \\
\hline G. arboreum & I & 149 & 238 & 19.8 & 0.3 & 2,546 & 34 & 67 & 2.1 & 0.1 & 9,046 \\
\hline G. raimondii & I & 142 & 379 & 18.9 & 0.5 & 1,599 & 29 & 66 & 1.8 & 0.1 & 9,183 \\
\hline G. hirsutum ${ }^{3}$ & 16 & 124 & 245 & 16.5 & 0.3 & 2,474 & 70 & 161 & 4.4 & 0.2 & 3,764 \\
\hline G. barbadense & 3 & 208 & 439 & 27.7 & 0.6 & $|, 38|$ & 48 & 117 & 3.0 & 0.2 & 5,180 \\
\hline G. mustelinum & I & 182 & 432 & 24.2 & 0.6 & 1,403 & 42 & 94 & 2.6 & 0.1 & 6,447 \\
\hline G. tomentosum & I & 156 & 382 & 20.8 & 0.5 & 1,587 & 34 & 84 & 2.1 & 0.1 & 7,215 \\
\hline Total & 24 & 270 & 1,005 & 36.0 & 1.3 & 603 & 92 & 279 & 5.8 & 0.4 & 2,172 \\
\hline
\end{tabular}

'Relative to 75 I single-copy loci

2Adjusted to average contig length of 807 bp

${ }^{3}$ Excluding $G$. hirsutum race yucatenense 
species polymorphism (Tables 2, 3 and 4). The SSCP prescreening and pooling strategy saved $77 \%$ of the resources compared to direct sequencing of individuals with 1607 amplifiable primers (Table 5).

Only amplicons showing polymorphism in pools were sequenced in individual lines. The germplasm panel included 16 G. hirsutum and three G. barbadense genotypes, plus tetraploids G. mustelinum and G. tomentosum and diploids G. arboreum and G. raimondii (Table 2). Sequencing of DNA templates resulted in different frequencies of SNPs and indels (Table 1). Sequences from 3' UTRs (252 bp/SNP), and SSR-associated (273 bp/SNP) sequences yielded 2-3 fold the frequency of SNPs than 5 'ends, COS, GSP and introns (464-807 bp/SNP). It is important to note that COS and GSP sequences contained introns. Similarly the frequency of indels was least in $5^{\prime}$ end sequences and greatest in 3' UTRs (Table 1). These results are consistent with those in maize showing that $3^{\prime}$ UTRs are a rich source of nucleotide diversity [26,27]. The lowest diversity in the 5 'ends is consistent with representation of more highly conserved coding sequences [19] . In comparison, the vacuolar H+-ATPase subunit [28] and Myb transcription factor families [29] were examined for diversity in diploid and tetraploid cotton species. The, 3' UTRs were 10-fold more polymorphic than coding sequences for Myb transcription factors [29].

Alternatively, designing primers from conserved singlecopy sequences (COS), or genome-specific primers (GSP) is an efficient method to access variation in introns for single loci in cotton. A closer examination at the different strategies indicates that GSP-based primers are less likely to amplify informative products than COS (60 vs. 91\%), but more likely to target single-locus sequences when they do (79 vs. $45 \%$ ). The majority of the COS sequences in our dataset were derived from diploid progenitors (see Materials and Methods). A COS-derived from tetraploid cotton only is likely to yield a larger proportion of singlecopy loci in tetraploids., Cotton intron regions were found to be less conserved than exons in the $\mathrm{H}^{+}$-ATPase subunit family [28]. Intron sequences have shown to be 3.7-fold more polymorphic than exons in cotton [20]. COS-based amplicons have also yielded a very high proportion of single-copy sequences (> 95\%) in Solanaceae $[19,30]$.

Another template target is to use predicted SNPs between diploid progenitor species to anchor primers to specific genomes (GSPs). Yang et al. [31] predicted 32,229 genome-specific SNPs from EST databases with $31 \%$ showing perfect concordance to the A or D genomes in the genotypes examined. In the current study, 3,000 SNPs were identified between only the diploid species and not within the tetraploid species (data not shown). As we sampled introns, many of these would be novel to ESTmined SNPs. Only 317 SNPs between G. arboreum and G. raimondii (Table 4 ) are polymorphic in $G$. hirsutum and $G$. barbadense. In our study, the estimates of diversity within and among species may not be representative of other species than G. hirsutum and G. barbadense, as only sequences that were polymorphic within and among these species were characterized. To ensure that single copy loci were being assayed, only SNPs that had clear homozygotes in at least one tetraploid were called.

\section{Diversity of cotton germplasm}

Within species diversity

SNPs were recorded as a base substitution relative to the consensus sequence derived from all genotypes. Although the number of individuals sampled varied, the speciesspecific diversity (as measured by the number of bases per

Table 4: Summary statistics for SNPs and indels between pairs of Gossypium species. The percentage is calculated out of total of 1005 SNPs and 279 indels.

\begin{tabular}{|c|c|c|c|c|c|c|c|c|c|c|c|}
\hline Number of SNPs & $G r^{\prime}$ & Gh & $G b$ & $G m$ & $G t$ & Number of indels & Gr & Gh & $G b$ & Gm & $G t$ \\
\hline G. arboreum & 317 & 303 & 201 & 334 & 261 & G. arboreum & 111 & 140 & 122 & 93 & 94 \\
\hline G. raimondii & & 452 & 430 & 266 & 317 & G. raimondii & & 169 & 153 & 84 & 115 \\
\hline G. hirsutum & & & 396 & 427 & 451 & G. hirsutum & & & 140 & 92 & 133 \\
\hline G. barbadense & & & & 396 & 448 & G. barbadense & & & & 91 & 113 \\
\hline G. mustelinum & & & & & 410 & G. mustelinum & & & & & 96 \\
\hline SNP Polymorphism (\%) & Gr & Gh & $G b$ & $G m$ & Gt & Indel Polymorphism (\%) & $G r$ & Gh & $G b$ & Gm & $G t$ \\
\hline G. arboreum & 32 & 30 & 20 & 33 & 26 & G. arboreum & 40 & 50 & 44 & 33 & 34 \\
\hline G. raimondii & & 45 & 43 & 26 & 32 & G. raimondii & & 61 & 55 & 3 & $4 I$ \\
\hline G. hirsutum & & & 39 & 42 & 45 & G. hirsutum & & & 50 & 33 & 48 \\
\hline G. barbadense & & & & 39 & 45 & G. barbadense & & & & 33 & $4 I$ \\
\hline G. mustelinum & & & & & 41 & G. mustelinum & & & & & 34 \\
\hline
\end{tabular}

$\mathrm{I}_{\mathrm{Gr}}=\mathrm{G}$. raimondii, $\mathrm{Gh}=\mathrm{G}$. hirsutum, $\mathrm{Gb}=\mathrm{G}$. barbadense, $\mathrm{Gm}=\mathrm{G}$. mustelinum, $\mathrm{Gt}=\mathrm{G}$. tomentosum 
Table 5: Summary of results for primer pairs tested in pools.

\begin{tabular}{lccc}
\hline Primer results & Number of primers & Percentage of preceding primer pool & Percentage of all primers tested \\
\hline Tested & 1974 & - & - \\
Amplified & 1607 & 41 & 81 \\
Single-copy & 751 & 49 & 38 \\
Polymorphic in pools & 365 & 96 & 18 \\
Successfully sequenced & 351 & 18 \\
\hline
\end{tabular}

IThirty-four single-copy loci were sequenced without pre-screening in pools.

SNP) was similar with two-fold lower frequency of SNPs for G. arboreum and G. hirsutum than the other species tested (Table 3, Additional File 1, Table S1). G. barbadense showed the highest level of diversity of the species tested with 1 SNP per 1381 bases and $28 \%$ of its loci being polymorphic. Conversely, G. hirsutum has the highest frequency of indels and the diploid progenitor species the least (Table 3, and Additional File 1, Tables S1 and S2). The average frequency of SNPs among $16 \mathrm{G}$. hirsutum lines was $1 / 2474 \mathrm{bp}(0.04 \%)$. In Adh genes, within G. hirsutum and G. barbadense diversity ranged from 1 to 3 SNPs in 983 bases $(0.1-0.3 \%)$ [32]. With the few reports of SNP frequencies in cotton, within G. hirsutum (3-4 lines), SNP frequency ranged from $0 / 1,000$ bp [29,32], 1/5,000 bp (0.02\%) [22] to $1 / 947 \mathrm{bp} \mathrm{(1 \% )} \mathrm{[21]} \mathrm{in} \mathrm{Adh,} \mathrm{Myb} \mathrm{and}$ expansin genes, respectively. Estimates of SNP divergence within and among species in the referenced studies were greater than those reported in our study for over 1,900 loci.

\section{Among species diversity}

SNP diversity in cotton has a large range that is both genome and gene specific. Compared to G. hirsutum, SNP frequency in G. arboreum, G. barbadense, G. mustelinum, G. tomentosum and $G$. raimondii ranged from $1 / 2,000$ bp $(0.05 \%)$ to $1 / 1,341 \mathrm{bp} / \mathrm{SNP}(0.075 \%)$ in order of increasing divergence (Table 4). Estimates among these species ranged from $1 / 51$ bp per SNP $(1.96 \%)$ in Myb and expansin genes between $G$. raimondii and $G$. hirsutum $[22,29]$ to $1 / 714$ bp per SNP $(0.14 \%)$ between G. hirsutum and $G$. barbadense in R2R3 Myb transcription factors $[21,33]$. The discrepancy in estimates is likely due to genespecific estimates, number of genes, germplasm used and the regions of the genes being sampled (coding vs. non coding). Non-coding sequence was reported to be 2-3 fold more polymorphic than coding regions $[21,22]$.

Comparisons of tetraploid species with G. raimondii (Dgenome) were consistently more divergent than those with G. arboreum (A-genome) except for G. mustelinum (Table 4). This agrees with observations using SSRs. ESTderived SSRs from diploid species tended to map more often to their orthologous genomes in corresponding tetraploid species [23], with G. raimondii-derived SSRs
(43\% polymorphism) being more polymorphic than $G$. arboreum derived SSRs (18\%) in the same cross [24,34]. Furthermore, in comparisons between A-genome diploids, a D-genome diploid, and tetraploids (G. hirsutum and G. barbadense), the rate of divergence in 48 genes was significantly higher in D-genomes than A-genomes, although the rate of divergence was gene-specific [33]. This was verified for specific gene families of transcription factors, Adh and expansins $[21,22,32]$ in cotton.

Several methods have been proposed for SNP discovery in allopolyploids and highly duplicated genomes including in silico analysis [31,35], genome specific-PCR [36], cloning and sequencing [29]. We have evaluated genome-specific amplification and high-throughput direct sequencing using M13-tailed amplicons combined with targeted primer design and pre-screening of primer pairs. We show that several options are feasible for high-throughput SNP discovery in cotton, each with their own advantages and disadvantages. The current research agrees with current literature that although tetraploid cottons were derived from a single polyploidization event from their diploid progenitors only 1-2 MYA [37], the genomes remain distinct and have sufficient diversity for breeding.

\section{Linkage Mapping}

SNPs were evaluated as markers by designing a 384-SNP array for the Illumina Golden Gate assay ${ }^{\otimes}$. Of the 384 SNPs on the array, 268 putative SNPs representing 240 contigs were expected to be polymorphic between the parents of our mapping population, TM-1 (G. hirsutum) and 3-79 (G. barbadense) and validated by assessing their segregation and amenability to linkage mapping. Of the 268 expected parental SNPs, 247 polymorphisms were detected using the Illumina assays on a population of 186 recombinant inbred lines (RILs) [23]. Segregation was as expected (1:1) for 188 markers, whereas 59 had skewed segregation resulting in 223 SNPs being placed on the linkage map (Figure 1). Markers that were not mapped had missing data or skewed segregation. The SNP markers are added to the TM-1/3-79 base map (Figure 1, and Yu et al in preparation) providing new tools for high-throughput genotyping in cotton. The SNP data are thus cross-referenced to several genetic $[6,8,14]$ and physical maps 
[38,39] via common SSRs (Yu et al in preparation). All SNPs, indels and flanking sequences can be accessed through the CMD database[16].

Although markers were evenly distributed within linkage groups, there was a disproportionate number of markers on linkage groups in the A subgenome (191 markers, $86 \%$ ) vs. the D subgenome (32 markers, $14 \%$; Figure 1, data not shown). At least $70 \%$ of the primers including those designed from 3 '-end, 5 '-end, intron and GSP, were derived exclusively from $G$. arboreum sequences, whereas the remainder were from G. arboreum, G. hirsutum and G. raimondii assemblies (see Materials and Methods). The fact that the majority of loci were derived from $G$. arboreum sequences and that these loci were in turn mapped to A-subgenome chromosomes in the TM-1 × 379 cross suggests that a) our approach for identifying single-locus markers was very effective; b) there is strong sequence conservation between the $\mathrm{A}_{2}$ genome of $G$. arboreum, and the A subgenomes of $G$. hirsutum ([AD $]_{1}$ ) and $G$. barbadense $\left([\mathrm{AD}]_{2}\right), \mathrm{c}$ ) the A and D subgenomes of contemporary $\mathrm{AD}$ genomes are distinct from each other; and d) the A subgenomes of G. hirsutum and G. barbadense are moderately divergent. The above conclusions are emphasized in the present study first by amplifying and sequencing single-copy loci and being able to assay the same sequences using an independent assay, Illumina Golden Gate. Because our template sequences were primarily drawn from G. arboreum and selected for both PCR function and single-locus attributes using SSCP, it is quite possible that the populations of selected loci in the two subgenomes would have been differentially affected. The potential for bias across the two subgenomes precludes using these amplicon sequence or genotyping data to infer relative diversity of the A versus D subgenomes of G. hirsutum and G. barbadense. Our findings are not discordant with the theory that these two extant tetraploids originated from a common allopolyploid ancestor and have evolved independently long enough to create the detected variation and a significant degree of diploidization.

\section{The use of SNPs in cotton}

SNP diversity depends on population size sampled and the natural evolutionary and directed selection within those populations. The present study indicates that a moderate amount of variation associated with genes exists in breeding germplasm (1 SNP in 2,474 bp in G. hirsutum) at the nucleotide level. G. barbadense germplasm sampled was 1.8 times as diverse (1 SNP in 1,381 bp) as G. hirsutum even though less than one fifth as many G. barbadense lines were sampled. The A and D genomes of tetraploid cotton are significantly divergent such that individual SNP loci can be assayed with high specificity. Although the above strategy for SNP identification and validation was fruitful, new high-throughput sequencing technologies such as 454 (Roche Biosciences, Branford, USA) Illumina Genome Analyzer (Hayward, USA) and SOLiD (Applied Biosystems, Foster City, USA) offer an opportunity to complement the current strategy to rapidly uncover nucleotide diversity at the whole genome level in multiple breeding lines. The present study demonstrates that sufficient SNP diversity exists in tetraploid cotton populations for genetic and breeding studies and it can be efficiently assayed.

\section{Conclusion}

In this research, SNP and indel diversity is characterized for 270 single-copy polymorphic loci in cotton. A strategy for SNP discovery is described to pre-screen loci for copy number and polymorphism. Diversity was characterized in a broad set of breeding lines and exotic lines representing a standard germplasm panel indicating that G. barbadense is much more diverse than G. hirsutum. Our data also indicate that the $\mathrm{A}$ and $\mathrm{D}$ genomes in both diploid and tetraploid cotton remain distinct from each other such that homoeologs can be distinguished. All marker data and flanking sequences have been submitted to Cotton Marker database http://www.cottonmarker.org/ Downloads.shtml

\section{Methods \\ Plant materials}

The 24 cotton lines and species screened for this study were chosen based on an expansion of the Cotton Marker Database (Table 2, [13]) standard germplasm panel assembled to represent the breadth of US cotton breeding germplasm and genetic standards. Consequently, the specific polymorphisms are expected to be relevant to these applications. The CMD panel has been genotyped with thousands of Simple Sequence Repeat (SSR) markers and serves as a resource to assess the utility of genetic markers in cotton. To address the low polymorphism in cotton breeding germplasm (11\%) [14] the CMD panel was expanded to include 12 additional elite breeding lines (Table 1). The panel contains crossing parents from G. tomentosum and G. mustelinum, and representation of the diploid species, G. arboreum and G. raimondii. As our main goal is to develop markers relevant to breeding germplasm 16 G. hirsutum and three G. barbadense (Pima) lines were selected. An additional landrace, TX2094, was added to represent G. hirsutum race yucatanense [40]. All accessions represent self-pollinating or inbred lines, thus should be homozygous for the majority of the loci.

\section{Primer design and screening}

To determine the optimum target template to identify SNPs specific to single-copy sequences in tetraploid cotton, we tested primer pairs from available BAC-end sequences; sequences flanking SSRs or predicted introns in ESTs; and sequences in the $3^{\prime}$ UTR or $5^{\prime}$ terminus of 

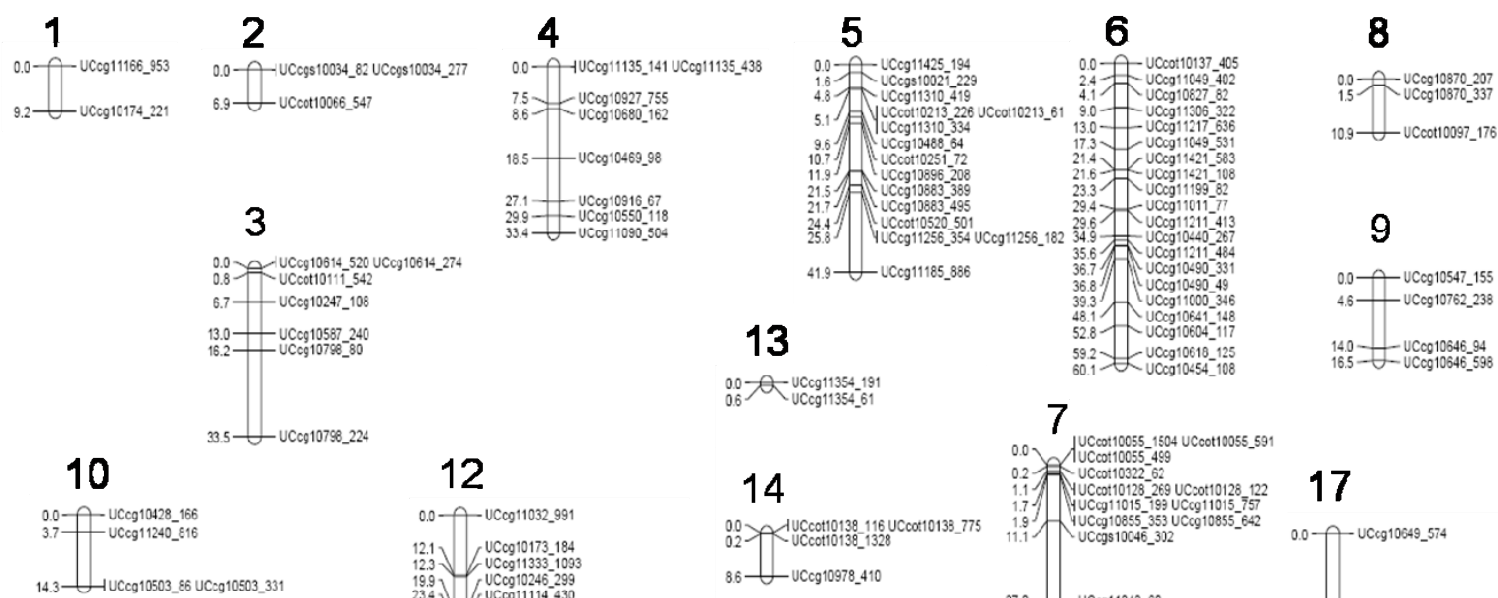

\section{2}

11
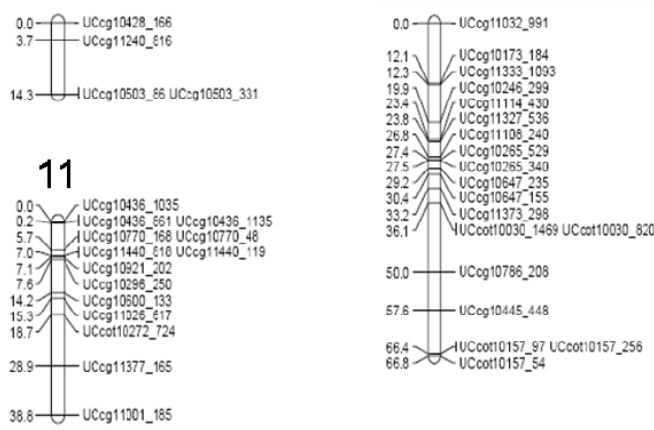

\section{3}

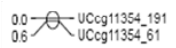

\section{4}

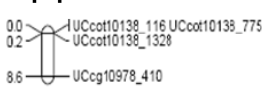

\section{5}
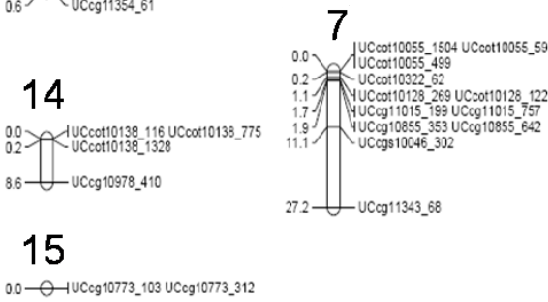

17

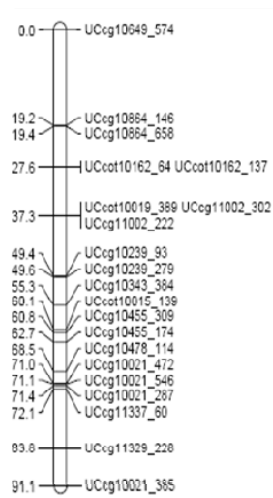

\section{6}
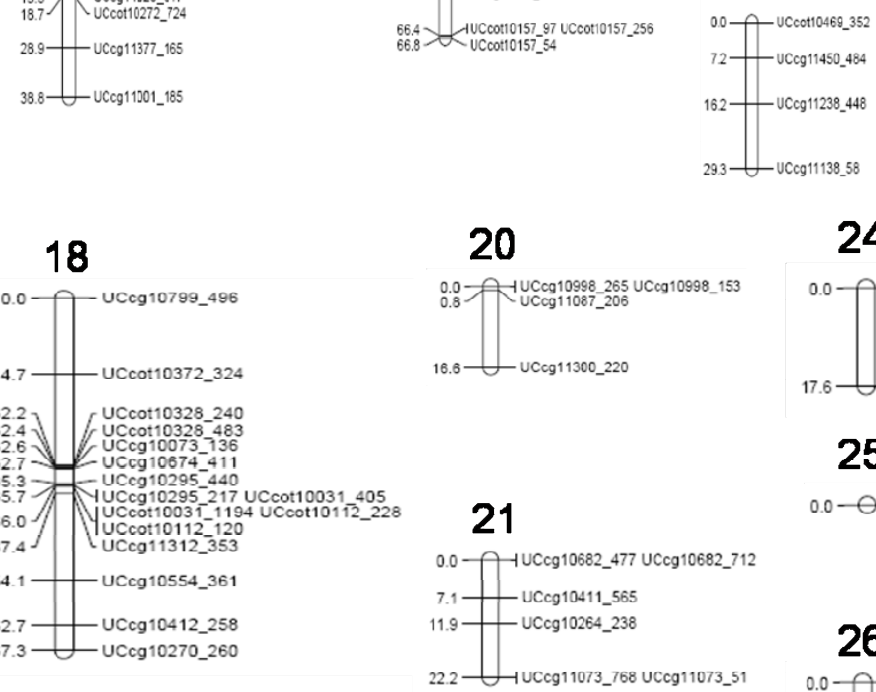

24

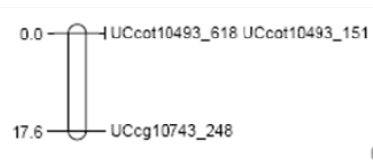

29

25

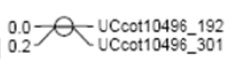

$0.0-\bigcirc-1 U C \cot 10393 \_208$ UCcot10393_558

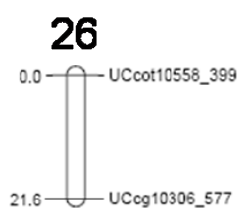

\section{0}

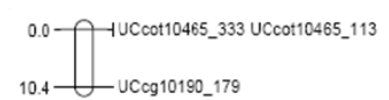

\section{9}

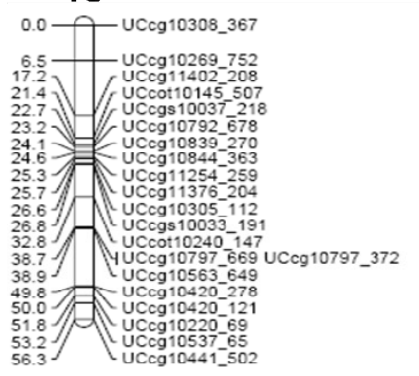

22

27

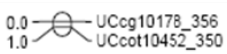

\section{3}

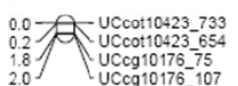

28 ${ }_{1.2}^{0.0}=-U$ UCcot10434 536

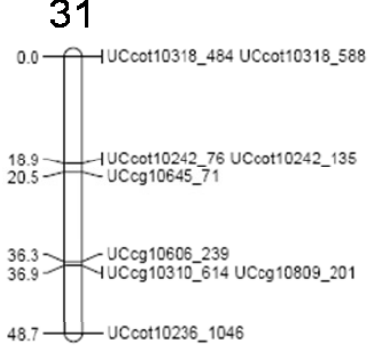

Figure I

Genetic map of 223 SNP markers in 186 recombinant inbred lines from a cross between TM-I and 3-79. Genetic distances in cM [46]. 
ESTs (Table 1). All sequences were downloaded from GenBank (Sept, 2004) except for SSR sequences kindly provided by Dr. Ben Burr, Brookhaven National Laboratories, NY). Based on the results, a database of primer sets with predicted product sizes of 600-800 bp was generated using Primer3 [41] to design primer pairs in the $3^{\prime}$ or $5^{\prime}$ termini of unigenes from G. arboreum ESTs [42]. As more ESTs became available, G. arboreum and G. raimondii ESTs were trimmed and assembled into 7,666 contigs with SNPs and deletions between these genomes. To evaluate the usefulness of cotton genome-specific primers, 48 primer pairs (GSP) were tested with a deletion in the forward primer and 48 with a SNP at the 3' end of the forward primer. A Conserved Orthologous Set for cotton of 2,390 contigs based on 27,878 G. arboreum, 35,509 G. raimondii and 14,354 G. hirsutum ESTs using the procedures described in Van Deynze et al (2007, [19]) was also generated as template for SNP discovery. A set of 576 primers (labeled with prefix of COT) was designed to amplify across predicted intron sites (based on Arabidopsis) with primers positioned 50-100 bp from the predicted introns

Two near-homozygous lines (G. arboreum and G. hirsutum breeding lines) were amplified and tested on agarose gels for amplification (Table 5). The amplified products were subsequently run on SSCP gels to screen for the presence of duplicated loci in amplification products. SSCP is a highly sensitive technique that detects variation in the nucleotide sequences of single-stranded molecules. Single-copy loci display two bands (the sense and antisense DNA strands). Loci displaying greater than two bands were not sequenced and were assumed to be not from a single locus. Primer pairs that successfully amplified a product and showed SSCP patterns of single-copy loci were tested for polymorphism using sequencing in a series of three pools representing different degrees of diversity in breeding germplasm: within elite G. hirsutum, a genetically diverse G. hirsutum, and between G. hirsutum and G. barbadense [19]. Each pool had three similar lines and one complementary genetically-distant line to maximize the chance of detecting a polymorphism within or among pools. Using a series of empirical tests with lines with known SNPs in ratios of 1:7, 1:5, 1:3 and 1:1, we determined that an unknown polymorphism can be reliably detected with sequencing with a 1:3 dilution. Pool 1 consisted of DPL458BR, Fibermax 832, Stoneville 4892BR, Sealand 5 42; Pool 2 consisted of PD1, Maxxa, Tamcot Sphinx, TX2094; and Pool 3 consisted of TM-1, 3-79, Pima S-7, Maxxa (Table 2). These pools represent increasing diversity in breeding germplasm. DNA was extracted from each line using Qiagen DNEASY (Qiagen, Valencia, USA) and was combined in equi-molar concentrations.

For all sequencing reactions, forward and reverse primers were tailed with M13 sequences and sequenced using standard protocols for Sanger sequencing (Applied Biosystems, Foster City, CA) in forward and reverse directions using a ABI 3730 (Applied Biosystems, Foster City, CA). Trace files were trimmed with Phred options -trim_cutoff 0.02" which translates to Phred 17 score [43]. Assembly was achieved with Phrap/Consed and options were set at "-retainduplicates and -forcelevel 5". These options were optimized to give the best trim and assembly parameters for calling SNPs. Stringent trim parameters are favored in this case to minimize the high number of false SNPs associated with poor sequence on the ends.

SNPs were first identified semi-manually using Polyphred as heterozygotes within pools or homozygous differences among pools. The line, HS200 (G. hirsutum), was used as reference to confirm that amplicons of single-copy loci were represented. Amplicons with putative SNPs were then amplified in the individual 24 lines (Table 2) and sequenced as described above. Only SNPs showing both homozygous alleles were called. Data was extracted from Polyphred using custom scripts ([44] See Supplemental files). Similarly, data for indels were extracted from Polyphred. Total polymorphism was calculated among genotypes and species as total number of SNPs, bases per SNP (SNP frequency) and percent polymorphic loci. Sequences are available through the Cotton Marker database [16].

A set of 384 SNPs was selected to develop an Illumina Golden Gate ${ }^{\circledast}$ oligonucleotide pooled assay. In order of priority, SNPs were selected to maximize the number of loci represented that were polymorphic between TM-1 and 3-79, or were polymorphic within G. hirsutum with moderate minor allele frequencies (>15\%) in G. hirsutum germplasm sequenced (Table 2). SNPs were genotyped in 186 RILs, the parents, and the $\mathrm{F}_{1}$ as per manufacturer protocols at the University of California, Genome and Biosciences Facility, Davis, CA. Data were extracted and exported and mapped using JoinMap 4.0 [45] with the Kosambi mapping function[46]. A LOD score of at least 6.0 was used to determine the linkage groups, of which the marker orders were verified at LOD score 3.0. Individual linkage groups were assigned to respective chromosomes by use of the TM-1 $\times 3-79$ base map (Yu et al, in preparation).

\section{List of abbreviations}

BAC: bacterial artificial chromosome; COS: conserved orthologous set; EST: expressed sequence tag; Indel: insertion/deletion; SNP: single nucleotide polymorphism

\section{Authors' contributions}

AV conceived, supervised and wrote the manuscript; KS carried out the research, contributed to methods and research and edited the paper; MK carried out the research, 
contributed to methods and research; AK helped conceive, analyse and edit the paper; TW aided in initiating the research, the EST database and edited the paper; RC inititated the research and developed the germplasm panel; JY contributed the mapping population, analysed the mapping data and edited the paper; RK contributed to the mapping population and analaysis; DS conceived the research, developed the germplasm panel, analyzed the data and edited the paper.

All authors have read and contributed to the writing of the manuscript.

\section{Additional material}

\section{Additional File 1}

Supplementary tables. Table S1. SNPs and associated sequence information. This worksheet describes the SNPs and the sequences they are derived from. Table S2. Indels and associated sequence information. This worksheet describes the SNPs and the sequences they are derived from. Table S3. Mapping data for TM-1 × 3-79 RIL population. This worksheet contains the data used for the mapping the SNPs. Table S4. Amplification and sequencing conditions used in this research. This worksheet describes the PCR protocol used to amplify and sequence genomic DNA.

Click here for file

[http://www.biomedcentral.com/content/supplementary/14712229-9-125-S1.XLS]

\section{Acknowledgements}

The authors would like to thank Mr. Brandon Tearse for converting raw polymorphism data to spreadsheet [44] format and Ms. Jessica Lund for technical assistance for generating the genotyping data. We would also like to thank Dr. Ben Burr (Brookhaven National Laboratory, NY, USA) for providing sequence data for SSRs. The authors would like to thank Cotton Incorporated and the University of California Discovery Grant for funding this research.

\section{References}

I. Bowman DT: Attributes of Public and Private Cotton Breeding Programs. J Cotton Science 2000, 4:130-136.

2. Robinson AF, Bell AA, Dighe ND, Menz MA, Nichols PL, Stelly DM: Introgression of resistance to nematode Rotylenchulus reniformis into upland cotton (Gossypium hirsutum) from Gossypium longicalyx. Crop Science 2007, 47(5): I865-I877.

3. He DH, Lin ZX, Zhang XL, Nie YC, Guo XP, Zhang YX, Li W: QTL mapping for economic traits based on a dense genetic map of cotton with PCR-based markers using the interspecific cross of Gossypium hirsutum $\times$ Gossypium barbadense. Euphytica 2007, I53(I-2): I81-197.

4. Zhang T, Yuan Y, Yu J, Guo W, Kohel RJ: Molecular tagging of a major QTL for fiber strength in Upland cotton and its marker-assisted selection. Theor Appl Genet 2003, I 06(2):262-268

5. Frary A, Fulton TM, Zamir D, Tanksley SD: Advanced backcross QTL analysis of a Lycopersicon esculentum $\times$ L. pennellii cross and identification of possible orthologs in the Solanaceae. Theoretical and Applied Genetics 2004, I 08(3):485-496.

6. Lacape JM, Nguyen TB, Thibivilliers S, Bojinov B, Courtois B, Cantrell RG, Burr B, Hau B: A combined RFLP-SSR-AFLP map of tetraploid cotton based on a Gossypium hirsutum $\times$ Gossypium barbadense backcross population. Genome/National Research
Council Canada $=$ Genome/Conseil national de recherches Canada 2003, 46(4):612-626.

7. Reinisch AJ, Dong JM, Brubaker CL, Stelly DM, Wendel JF, Paterson $\mathrm{AH}$ : A detailed RFLP map of cotton, Gossypium hirsutum $\times$ Gossypium barbadense: chromosome organization and evolution in a disomic polyploid genome. Genetics 1994, I 38(3):829-847.

8. Rong J, Abbey C, Bowers JE, Brubaker CL, Chang C, Chee PW, Delmonte TA, Ding X, Garza J], Marler BS, Park CH, Pierce GJ, Rainey KM, Rastogi VK, Schulze SR, Trolinder NL, Wendel JF, Wilkins TA, Williams-Coplin TD, Wing RA, Wright RJ, Zhao X, Zhu L, Paterson $\mathrm{AH}$ : A 3347-locus genetic recombination map of sequencetagged sites reveals features of genome organization, transmission and evolution of cotton (Gossypium). Genetics 2004, I 66(I):389-4I7.

9. Waghmare VN, Rong JK, Rogers CJ, Pierce GJ, Wendel JF, Paterson $\mathrm{AH}$ : Genetic mapping of a cross between Gossypium hirsutum (cotton) and the Hawaiian endemic, Gossypium tomentosum. Theoretical and Applied Genetics 2005, I I I (4):665-676.

10. Chen Z]: Genetic and epigenetic mechanisms for gene expression and phenotypic variation in plant polyploids. Annual review of plant biology 2007, 58:377-406.

II. Rong J, Pierce G], Waghmare VN, Rogers C], Desai A, Chee PW, May OL, Gannaway JR, Wendel JF, Wilkins TA, Paterson AH: Genetic mapping and comparative analysis of seven mutants related to seed fiber development in cotton. Theor Appl Genet 2005: I- 10 .

12. Guo W, Cai C, Wang C, Han Z, Song X, Wang K, Niu X, Lu K, Shi B, Zhang T: A microsatellite-based, gene-rich linkage map reveals genome structure, function and evolution in Gossypium. Genetics 2007, I 76(I):527-54I.

13. Blenda A, Scheffler J, Scheffler B, Palmer M, Lacape JM, Yu JZ, Jesudurai $C$, Jung $S$, Muthukumar S, Yellambalase P, Ficklin S, Staton M, Eshelman R, Ulloa M, Saha S, Burr B, Liu S, Zhang T, Fang D, Pepper A, Kumpatla S, Jacobs J, Tomkins J, Cantrell R, Main D: CMD: a Cotton Microsatellite Database resource for Gossypium genomics. BMC Genomics 2006, 7:I32.

14. Frelichowski JE, Palmer MB, Main D, Tomkins JP, Cantrell RG, Stelly $\mathrm{DM}, \mathrm{Yu}$ J, Kohel RJ, Ulloa M: Cotton genome mapping with new microsatellites from Acala 'Maxxa' BAC-ends. Molecular Genetics and Genomics 2006, 275(5):479-49I.

15. Volker B, Lushbough C, Lawrence C: PlantGDB: Resources for Plant Comparative Genomics. [http://www.plantgdb.org/prj/ ESTCluster/progress.php].

16. Blenda A, Van Deynze A, Main D: CMD: a Cotton Microsatellite Database resource for Gossypium genomics. UC Davis SNP project. [http://www.cottonmarker.org/Downloads.shtml].

17. Rungis D, Llewellyn D, Dennis ES, Lyon BR: Simple sequence repeat (SSR) markers reveal low levels of polymorphism between cotton (Gossypium hirsutum L.) cultivars. Australian Journal of Agricultural Research 2005, 56(3):30 I-307.

18. Van Becelaere G, Lubbers EL, Paterson AH, Chee PW: Pedigree- vs. DNA marker-based genetic similarity estimates in cotton. Crop Science 2005, 45(6):228I-2287.

19. Van Deynze AE, Stoffel K, Buell RC, Kozik A, Liu J, Knaap E van der, Francis D: Diversity in conserved genes in tomato. BMC Genomics 2007, 8(I):465.

20. Chee PW, Rong J, Williams-Coplin D, Schulze SR, Paterson AH: EST derived PCR-based markers for functional gene homologues in cotton. Genome/National Research Council Canada = Genome/Conseil national de recherches Canada 2004, 47(3):449-462.

21. An C, Saha S, Jenkins JN, Ma DP, Scheffler BE, Kohel RJ, Yu JZ, Stelly DM: Cotton (Gossypium spp.) R2R3-MYB transcription factors SNP identification, phylogenomic characterization, chromosome localization, and linkage mapping. Theor Appl Genet 2008, I 16(7): 1015-1026.

22. An C, Saha S, Jenkins JN, Scheffler BE, Wilkins TA, Stelly DM: Transcriptome profiling, sequence characterization, and SNPbased chromosomal assignment of the EXPANSIN genes in cotton. Mol Genet Genomics 2007, 278(5):539-553.

23. Park YH, Alabady MS, Ulloa M, Sickler B, Wilkins TA, Yu J, Stelly DM, Kohel RJ, el-Shihy OM, Cantrell RG: Genetic mapping of new cotton fiber loci using EST-derived microsatellites in an interspecific recombinant inbred line cotton population. Mol Genet Genomics 2005, 274(4):428-44I. 
24. Wang CB, Guo WZ, Cai CP, Zhang TZ: Characterization, development and exploitation of EST-derived microsatellites in Gossypium raimondii Ulbrich. Chinese Science Bulletin 2006, 5I(5):557-56I.

25. Zhang J, Pang MX, Niu C, Wang W, Percy RG, Cantrell RG, Stewart JM: AFLP-Based SNP Discovery In Cotton. Plant \& Animal Genomes XV Conference. San Diego, CA 2007.

26. Eveland AL, McCarty DR, Koch KE: Transcript profiling by 3'untranslated region sequencing resolves expression of gene families. Plant physiology 2008, I 46(I):32-44.

27. Ching A, Caldwell KS, Jung M, Dolan M, Smith OS, Tingey S, Morgante $M$, Rafalski A): SNP frequency, haplotype structure and linkage disequilibrium in elite maize inbred lines. BMC Genet 2002 3(1): 19.

28. Wilkins TA, Wan CY, Lu CC: Ancient Origin of the Vacuolar H+-Atpase 69-Kilodalton Catalytic Subunit Superfamily. Theoretical and Applied Genetics 1994, 89(4):5। 4-524.

29. Hsu CY, An C, Saha S, Ma DP, Jenkins JN, Scheffler B, Stelly DM: Molecular and SNP characterization of two genome specific transcription factor genes GhMyb8 and GhMybl0 in cotton species. Euphytica 2008, I 59( I-2):259-273.

30. Wu FN, Mueller LA, Crouzillat D, Petiard V, Tanksley SD: Combining bioinformatics and phylogenetics to identify large sets of single-copy orthologous genes (COSII) for comparative, evolutionary and systematic studies: $A$ test case in the euasterid plant clade. Genetics 2006, I74(3):|407-|420.

31. Yang SS, Cheung F, Lee JJ, Ha M, Wei NE, Sze SH, Stelly DM, Thaxton $P$, Triplett B, Town CD, Jeffrey Chen Z: Accumulation of genome-specific transcripts, transcription factors and phytohormonal regulators during early stages of fiber cell development in allotetraploid cotton. Plant J 2006, 47(5):76I-775.

32. Small RL, Ryburn JA, Wendel JF: Low levels of nucleotide diversity at homoeologous Adh loci in allotetraploid cotton (Gossypium L.). Molecular biology and evolution 1999, 16(4):49I-50I.

33. Senchina DS, Alvarez I, Cronn RC, Liu B, Rong J, Noyes RD, Paterson AH, Wing RA, Wilkins TA, Wendel JF: Rate variation among nuclear genes and the age of polyploidy in Gossypium. Molecular biology and evolution 2003, 20(4):633-643.

34. Han ZG, Guo WZ, Song XL, Zhang TZ: Genetic mapping of ESTderived microsatellites from the diploid Gossypium arboreum in allotetraploid cotton. Mol Genet Genomics 2004 272(3):308-327.

35. Udall JA, Swanson JM, Haller K, Rapp RA, Sparks ME, Hatfield J, Yu Y, Wu Y, Dowd C, Arpat AB, Sickler BA, Wilkins TA, Guo JY, Chen XY, Scheffler J, Taliercio E, Turley R, McFadden H, Payton P, Klueva N, Allen R, Zhang D, Haigler C, Wilkerson C, Suo J, Schulze SR, Pierce ML, Essenberg M, Kim H, Llewellyn DJ, Dennis ES, Kudrna D, Wing R, Paterson $\mathrm{AH}$, Soderlund $\mathrm{C}$, Wendel JF: A global assembly of cotton ESTs. Genome Res 2006, 16(3):44I-450.

36. Caldwell KS, Dvorak J, Lagudah ES, Akhunov E, Luo MC, Wolters P, Powell W: Sequence polymorphism in polyploid wheat and their D-genome diploid ancestor. Genetics 2004, 167(2):941-947.

37. Wendel JF, Cronn RC: Polyploidy and the evolutionary history of cotton. Advances in Agronomy 2003, 78: |39-186.

38. Gao W, Chen ZJ, Yu JZ, Raska D, Kohel RJ, Womack JE, Stelly DM Wide-cross whole-genome radiation hybrid mapping of cotton (Gossypium hirsutum L.). Genetics 2004, 1 67(3): 13 | 7 - 1329.

39. Liu S, Saha S, Stelly D, Burr B, Cantrell RG: Chromosomal assignment of microsatellite loci in cotton. Journal of Heredity 2000, 9I(4):326-332.

40. Liu S, Cantrell RG, McCarty JC, Stewart JM: Simple sequence repeat-based assessment of genetic diversity in cotton race stock accessions. Crop Science 2000, 40(5): | 459-| 469.

4I. Rozen S, Skaletsky H: Primer3 on the WWW for general users and for biologist programmers. Methods in molecular biology (Clifton, NJ) 2000, I32:365-386.

42. Arpat A, Waugh M, Sullivan J, Gonzales M, Frisch D, Main D, Wood T, Leslie A, Wing R, Wilkins T: Functional genomics of cell elongation in developing cotton fibers. Plant Molecular Biology 2004 54(6): $911-929$.

43. Ewing B, Green P: Base-calling of automated sequencer traces using phred. II. Error probabilities. Genome research 1998, 8(3): $186-194$.

44. Tearse B: Polyphred to Excel converter. [http://den drome.ucdavis.edu/resources/].
45. Van Ooijen JW: JoinMap 4.0, Software for the calculation of genetic linkage maps in experimental populations. Wageningen, Netherlands: Kyazma B.V; 2006.

46. Kosambi DD: The estimation of map distances from recombination values. Ann Eugen 1944, I 2: 172-175.
Publish with Bio Med Central and every scientist can read your work free of charge

"BioMed Central will be the most significant development for disseminating the results of biomedical research in our lifetime. "

Sir Paul Nurse, Cancer Research UK

Your research papers will be:

- available free of charge to the entire biomedical community

- peer reviewed and published immediately upon acceptance

- cited in PubMed and archived on PubMed Central

- yours - you keep the copyright 